\title{
Diagnostic difficulties in chronic obstructive pulmonary disease exacerbations: A case report
}

\author{
Simona Stefania Bucsa ${ }^{1^{*}}$, Petre lacob Calistru ${ }^{2}$ \\ ${ }^{1}$ Department of Pneumology, “Dr Victor Babes” Diagnosis and Treatment Center, Bucharest, Romania; \\ *Corresponding Author: simonabucsa@yahoo.com \\ ${ }^{2}$ Department of Infectious Diseases, Faculty of Medicine, Carol Davila University of Medicine and Pharmacy, Bucharest, Romania
}

Received 16 May 2013; revised 10 June 2013; accepted 30 June 2013

Copyright (c 2013 Simona Stefania Bucsa, Petre Iacob Calistru. This is an open access article distributed under the Creative Commons Attribution License, which permits unrestricted use, distribution, and reproduction in any medium, provided the original work is properly cited.

\section{ABSTRACT}

Exacerbations of COPD (chronic obstructive pulmonary disease) influence, by their frequency and severity, the life prognosis of patients with COPD. Most exacerbations are caused by respiratory infections with negative impact on patient quality of life. The impact of frequent exacerbation in COPD is manifested by the rapid decline of lung function, decreased quality of life increased airway inflamation and high mortality. Bronchial obstruction by foreign bodies meets frequently during childhood but can occur in adults under certain conditions of loss of consciousness (sleep, administration of tranquilizers, after anesthesia, intoxicated). The longstanding intrabronchial foreign body presents problems of diagnosis and treatment which are very different from those associated with the recently inhaled foreign body. We herein report a 77-year-old male, smoker (40 pack-year) presented to the clinic with severe dyspnoea, fever and cough with purulent sputum for about 6 months. Persistent symptoms require repeated hospitalizations for receiving different regimens of antibiotics, but they have not helped. Chest radiography showed extensive consolidation of the right lower lobe, intensity rib, heterogeneous and Chest CT scan showed metallic foreign body in right lower lobar bronchus with secondary pneumonic process in the lower right lobe. Emergency was performed bronchoscopy and extracted dental crown consists of three teeth and then the patient received combined antibiotic therapy for 14 days, in association with anti-inflammatory, mucolytics and bronchodilators. After 2 weeks he was overall in very good condition and all other complaints disappeared and Chest X-ray control showed full resorption of pneumonia opacity, without disabling signs. In this particular case, only minor symptoms are seen at the beginning and the aspirated foreign body was forgotten until later symptoms (inflammation, infection) were developed and developing clinically manifest. The diagnosis was delayed due to lack of radiographic view which may be aspirated foreign body embedded in granulation tissue formed around.

Keywords: Retrostenotica Secondary Pneumonia; Metallic Foreign Body Aspirated Intrabronsic

\section{INTRODUCTION}

Bronchial obstruction by foreign bodies (FB) meets frequently during childhood, and commonly after the age of 1 year to 45 years. The highest incidence occurs between the age of 1 - 3 years that is $77 \%$ [1] and it is rare in adults. Aspiration of FB is multifactorial in their aetiology, in their broad spectrum of different resolutions for the same FB and in the response of each patient to the treatment.

In aspiration of foreign body symptoms are three clinical phases: initial stage (first stage or impaction or FB) showing choking, gagging and paroxysms of coughing, obstruction of the airway (AW), occurring at the time of aspiration. These signs calm down when the FB lodges and the reflexes grow weary (second stage or asymptomatic phase).

Complications occur in the third stage (also defined as the complication phase), when the obstruction, erosion or infection cause pneumonia, atelectasis, abscess or fever. The first symptoms to receive medical care may actually represent a complication of impaction of FB [2]. Early 
diagnosis and treatment is imperative to prevent mortality as well as complications.

The long-standing intrabronchial foreignbody presents problems of diagnosis and treatment which are very different from those associated with the recently inhaled foreign body. There may be no history to suggest the original incident; the clinical picture is usually clouded by superadded pathological changes-atelectasis, pneumonitis, bronchiectasis, or lung bscess-and the bronchoscopic appearances are often misleading. The symptoms of aspiration of FB can simulate different diseases such as asthma, croup or pneumonia, delaying the correct diagnosis [2]. The delay in the removal of FB is potentially harmful. Even when correctly diagnosed, the choice of treatment is not always easy. The treatment of choice for AW is endoscopic removal [2].

Bronchoscopy may be necessary to make a definitive diagnosis and to remove the object. Prevention remains the best treatment, but in case of complications antibiotics and respiratory therapy techniques may be used if infection develops. Sometimes, for the removal of highly difficult and high risk tracheobronchial foreign bodies, preoperative analysis and discussion should be sufficient, appropriate surgical skill and surgical instruments may improve the success rate of the surgery and prevent the operation complications [3].

\section{CASE REPORT}

A 77-year-old male, smoker (40 pack-year), living in villages, presented to the clinic with severe dyspnoea, fever and cough with purulent sputum for about 6 months. He has a history of Chronic Obstructive Pulmonary Disease (COPD) 5 years ago, with two exacerbations in the past year, diabetes mellitus with oral treatment and chronic use of ethanol. Current disease onset was 6 months ago with cough with purulent sputum, intermitent fever and chills, shortness of breath. He was hospitalized in the village with diagnosis of COPD exacerbations, community acquired pneumonia and received antibiotic treatment with Amoxicilin-Clavulanat and Gentamicin, then Ceftriaxon, with temporary improvement. Then recurrence the simptoms determined repeated hospitalizations for which he received different antibiotic regimens, but they have not helped. A few days before presenting to hospital , the symptoms worsened, the patient presenting malaise, severe dyspnoea with weezing, fever and cough with purulent sputum. Physical examination on admission: General appearance: underweight; Respirator stetacustic. prolonged expiration, weezing, bronchial rales crackles in lower right lung, $\mathrm{SaO}_{2}=91 \%$ - 93\%; Febrile, $\mathrm{T}=38.5^{\circ} \mathrm{C}$; Vital signs: in normal limits; No other significant signs revealed. Blood tests showed: leucocytes $17.800 / \mathrm{mm}^{3}$, with neutrophils $9.900 / \mathrm{mm}^{3}$; $\mathrm{VSH}=68 / 105 \mathrm{~mm} / \mathrm{h} / 2 \mathrm{~h} ; \mathrm{CRP}=8.68 \mathrm{mg} / \mathrm{dl}$; Fibrinogen
= 785 mg/dl; TGP, TGO, Glycemia, BUN within normal ranges; specific tumor markers-negative. Chest radiography showed extensive consolidation of the right lower lung lobe,intensity rib, heterogeneous (Figure 1). Spirometry: severe mixed ventilatory dysfunction with reduced $\mathrm{FEV}_{1}$ with $73 \%(\mathrm{FEV} 1=27 \%$, FVC= 63\%, Tiffeneau Index = 34.6) (Figure 2). Abdominal ultrasound, cardiac ultrasound, all in normal limits. We performed a Chest CT scan contrast enhanced which showed metallic foreign body in right lower lobar bronchus with secondary pneumonic process in the lower right lobe (Figures 3(a) and (b)).

The diagnosis is: Intrabronchial foreign body with airway obstruction secondary pneumonia, COPD group D. Emergency bronchoscopy was performed. Bronchoscopy showed that the mucosa of the right lower lobe was hyperaemic, granular, much pus and extracted a dental crown made of three teeth. Than we started antibiotics treatment with Amoxicilin-Clavulanat $1 \mathrm{~g}$ three times a day and Metronidazol $1 \mathrm{~g}$ twice a day for 14 days in association with anti-inflammatory, mucolytics and bronchodilators (Corticosteroid inhaled with Long action $\beta$ agonist fixed combinations). With tratment and postural drainage, he became afebrile in three days, general condition improved and than the cough and dyspnoea improved.

Treated, after 2 weeks he was overall very good condition and all other complaints disappeared. Control Chest X-ray showed complete resolution of pneumonia opacity without other signs added (Figure 4).

\section{DISCUSSION}

Although FB aspiration can occur at any stage in life, it is far more common in children. In adults, FB aspiration is uncommon and is mostly related to accidental aspiration of working tools (paper clips, tacks, or nails)

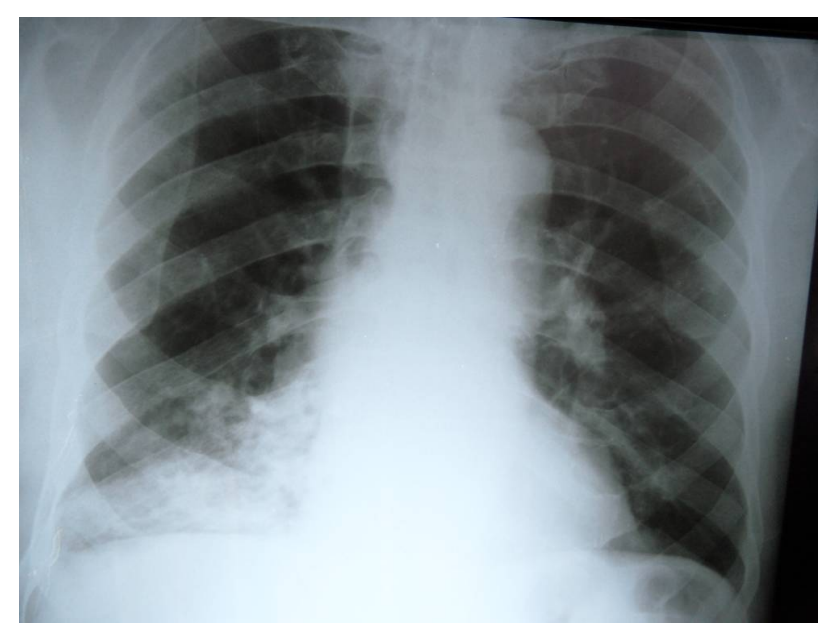

Figure 1. Pretreatment chest X-ray posterior-anterior shows extensive consolidation in the right lower lung lobe. 


\section{CENTRUL DE DIAGNOSTIC SI TRATAMENT "Dr. V. BABES"}

Adresa: Sos. Mihai Bravu,Nr.281, Bucuresti, Sect 3

Tel: 021.3179503; 021.3179504; 021.3179505

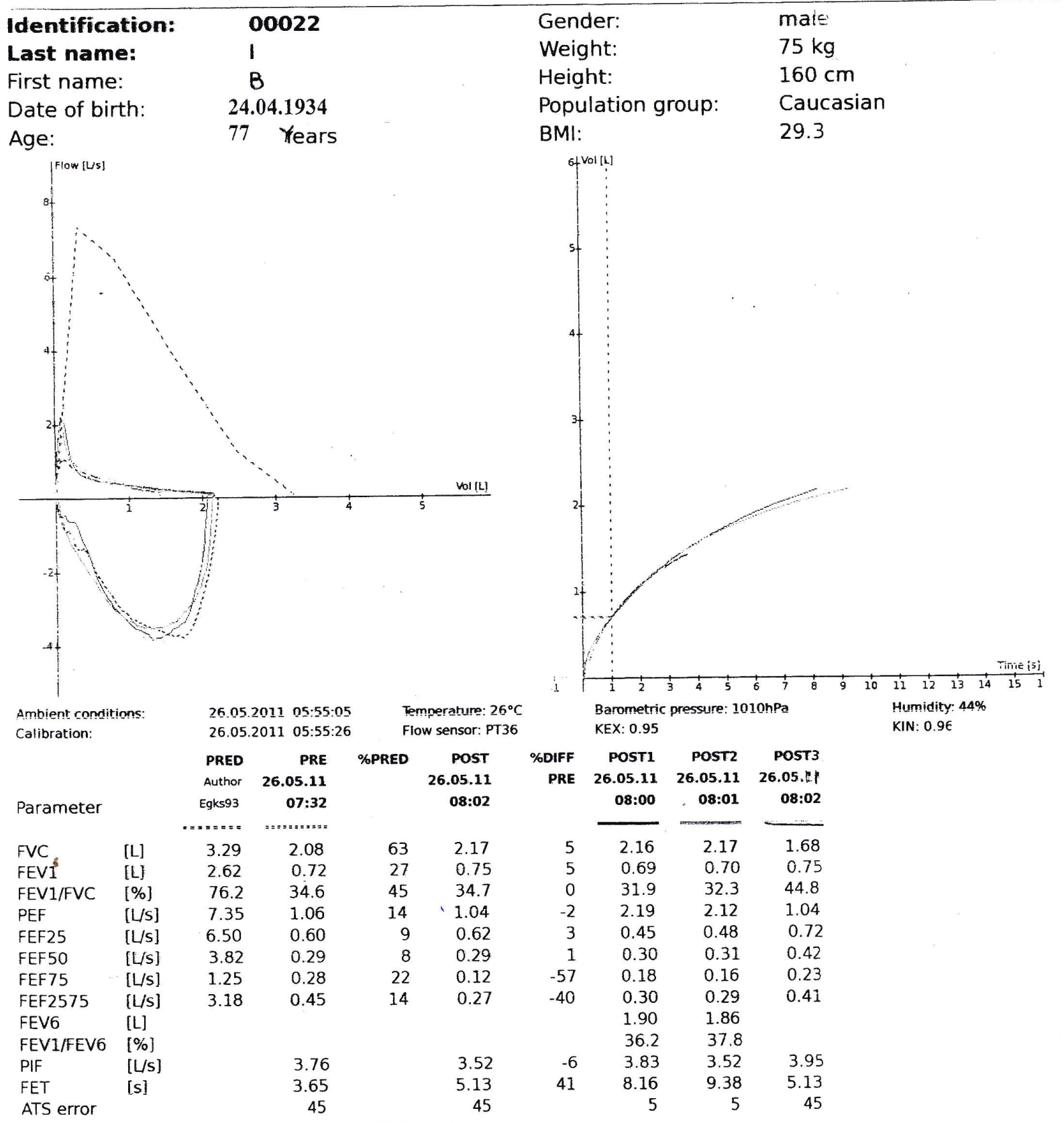

ATS legend: (4) Expiratory time too short. (5) No plateau.

Medication: Ventolin $0.96 \mathrm{mcg}$

\section{interpretation help: ( Europe)}

Severe expiratory flow limitation. Additional examination recommended to exclude extra-thoracic flow limitation and expiratory tracheal stenosis. Pay attention to patient cooperation. Moderate to severe expiratory flow limitation during deep exhalation. Additional pulmonary function measurement recommended

Reversibility Check: Not reversible or hardly susceptible obstruction.

JAEGER FlowScreen $B$ V2.2.2 Serial number: 38210222

Figure 2. Spirometry show severe mixed ventilatory dysfunction with reduced $\mathrm{FEV}_{1}$ with $73 \%(\mathrm{FEV} 1=27 \%, \mathrm{FVC}=63 \%$, Tiffeneau Index = 34.6). 


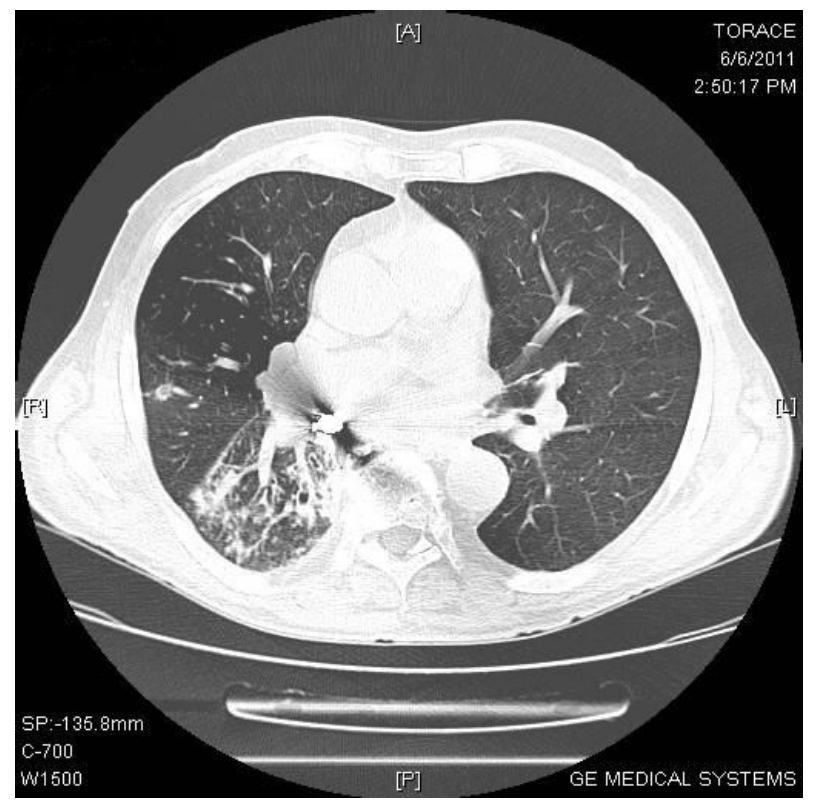

(a)

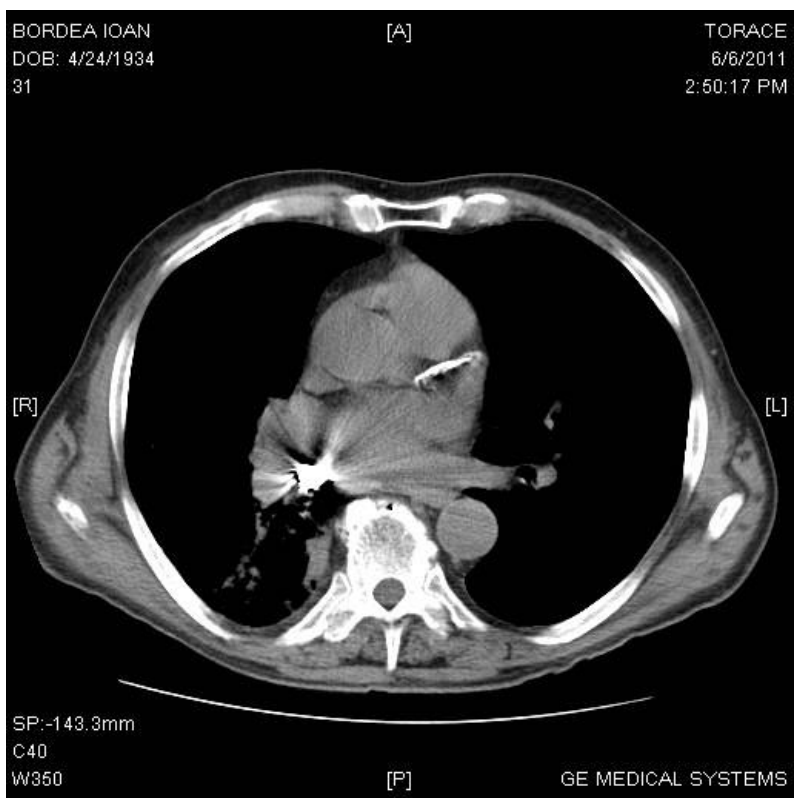

(b)

Figure 3. (a), (b) CHEST CT scan findings metallic foreign body in right lower lobar bronchus with secondary pneumonic process in the lower right lobe.

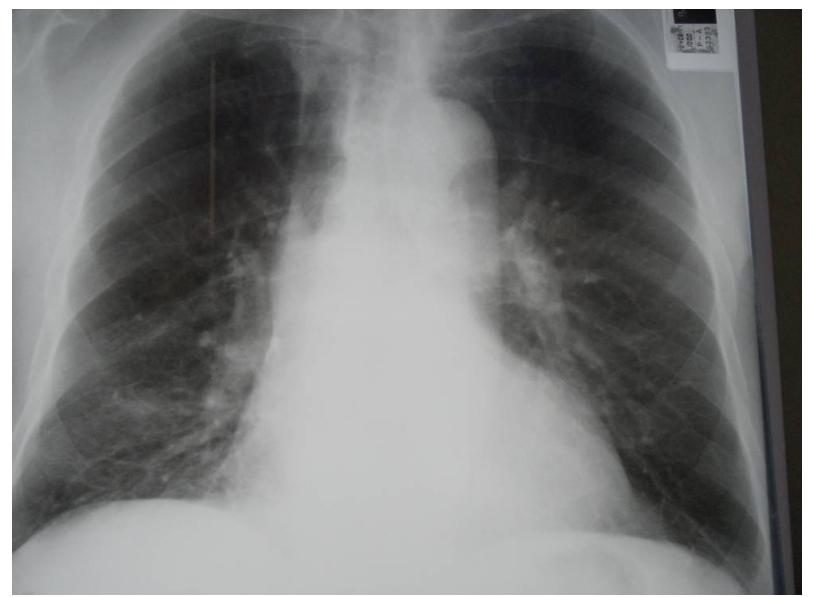

Figure 4. After treatment chest X-ray showed complete resolution of pulmonary opacity without other signs added.

and bone fragments, as well as occurring during unconsciousness (trauma, general anesthesia, sedation, intoxication, seizures, and neurological disorders) [1].

Clinical onset is sudden, often impressive and sometimes with rapidly fatal evolution. However, in some cases, only minor symptoms are seen at first, and the object may be forgotten until later symptoms (inflammation, infection) develop. After overcoming the acute episode, the patient may remain asymptomatic for a period then symptoms debilitating chronic recurrent infections may delay late foreign body extraction. In the case of retained foreign bodies, the possibilities of granulation tissue and post-obstruction infection exist. The accurate diagnosis may be missed even by an experienced clini- cian because often the initial choking episode is not witnessed and also the delayed symptoms may mimic other common conditions like asthma, COPD exacerbations, recurrent pneumonia, upper respiratory infection and persistent cough. Diagnosis and removal of an inhaled foreignbody are required as quickly as possible in order to preven mortality as well as complications.

In the present case, the symptoms did not appear immediately after bronchial obstruction and were hidden with antibiotics and physiotherapy symptomatic treatment but were repeated at intervals until the foreign body was finally removed. Diagnostic imaging plays a variable role in identifying airways foreign bodies.Most of the foreign bodies are not radiopaque and small foreign bodies may cause symptoms but no radiographic signs. Plain films may be inadequate to document a non radioopaque foreign body unless they are obtained in the expiratory phase. Rodrigues AJ and colleagues showed in their study that $25 \%$ of the chest $\mathrm{X}$-ray findings were normal, despite a clinical history of FB aspiration [4].

Regardless of the equipment used, bronchoscopy should be performed in all cases of suspected FB aspiration, even if the radiological findings are normal [5,6]. Bronchoscopy remains the gold standard for the diagnosis and treatment of FB aspiration [7]. Virtually all aspirated FBs can be extracted by bronchoscopy, with success rates above $98 \%$. Rigid bronchoscopy remains the standard procedure for the removal of $\mathrm{FB}$ [8] and has various advantages over flexible bronchoscopy. Rigid bronchoscopes are larger in diameter than being flexible bronchoscopes, therefore allowing blood aspiration, thick 
secretion aspiration, and patient ventilation.

Although rigid bronchoscopy is considered the gold standard for the removal of foreign bodies from the airways, exist studys that demonstrated that flexible bronchoscopy can be safely and effectively used in the diagnosis and treatment of stable adult patients [4].

In the rare instances in which bronchoscopic removal fails, surgical bronchotomy or segmental resection is indicated. The presence of chronic bronchial obstruction, together with bronchiectasis, lung abscess, and parenchymal destruction, might be an indication for segmental or lobar resection [9].

In this particular case, the diagnosis was delayed due to lack of radiographic visualization of the foreign body intrabronsic with persistent symptoms of infection despite antibiotic treatment.

\section{ACKNOWLEDGEMENTS}

This paper is supported by the Sectoral Operational Programme Human Resources Development (SOP HRD) 2007-2013, financed from the European Social Fund and by the Romanian Government under the contract number POSDRU/107/1.5/S/82839.

\section{REFERENCES}

[1] Qureshi, A. and Behzadi, A. (2008) Foreign-body aspiration in an adult. Canadian Journal of Surgery, 51, E69E70.

[2] Rodríguez, H., Passali, G.C., Gregori, D., Chinski, A., Tiscornia, C., Botto, H., Nieto, M., Zanetta, A., Passali, D. and Cuestas, G. (2012) Management of foreign bodies in the airway and oesophagus. International Journal of Pediatric Otorhinolaryngology, 76, S84-S91.

\section{doi:10.1016/j.ijporl.2012.02.010}

[3] Xu, E.M., Xu, Z.Q., Wang, Z.N., Wang, Y., Chen, P. Zhang, Y.M., Xia, F and Zhang, Y. (2012) Experience in the removal of difficult and high risk trachea bronchial foreign body by bronchoscopy. Chinese Journal of Otorhinolaryngology Head and Neck Surgery, 47, 982-986.

[4] Rodrigues, A.J., Oliveira, E.Q., Scordamaglio, P.R., Gregório, M.G., Jacomelli, M. And Figueiredo, V.R. (2012) Flexible bronchoscopy as the first-choice method of removing foreign bodies from the airways of adults. Jornal Brasileiro de Pneumologia, 38, 315-320. doi:10.1590/S1806-37132012000300006

[5] Orji, F.T. and Akpeh, J.O. (2010) Tracheobronchial foreign body aspiration in children: How reliable are clinical and radiological signs in the diagnosis? Clinical Otolaryngology, 35, 479-485. doi:10.1111/j.1749-4486.2010.02214.x

[6] Korlacki, W., Korecka, K. and Dzielicki, J. (2011) Foreign body aspiration in children: Diagnostic and therapeutic role of bronchoscopy. Pediatric Surgery International, 27, 833-837. doi:10.1007/s00383-011-2874-8

[7] Shlizerman, L., Mazzawi, S., Rakover, Y. and Ashkenazi, D. (2010) Foreign body aspiration in children: The effects of delayed diagnosis. American Journal of Otolaryngology, 31, 320-324. doi:10.1016/j.amjoto.2009.03.007

[8] Grover, S., Bansal, A. and Singhi, S.C. (2011) Airway foreign body aspiration. Indian Journal of Pediatrics, 78, 1401-1403. doi:10.1007/s12098-011-0488-8

[9] Isherwood, J. and Firmin, R. (2011) Late presentation of foreign body aspiration requiring extracorporeal membrane oxygenation support for surgical management. Interactive CardioVasc Thoracic Surgery, 12, 631-632. 